\title{
SELF-ORDERED COLLOIDAL ARRAYS AS PHOTONIC CRYSTAL HYDROGELS FOR TRAINABLE METAL ION SENSORS AND AS SUPERPARAMAGNETIC MATERIALS
}

\author{
ANJAL C. SHARMA*, XIANGLING XU*, MICHELLE S. WARD*, LONG LI**, JUDITH C. \\ YANG** AND SANFORD A. ASHER* \\ *Department of Chemistry, 234 Chevron Science Center, University of Pittsburgh, Pittsburgh, PA \\ 15260. \\ **Department of Materials Science and Engineering, 848 Benedum Hall, University of Pittsburgh, \\ Pittsburgh, PA 15260.
}

We have developed novel materials based on colloidal self-assembly, which are ordered arrays of colloidal particles formed in a liquid. We have polymerized these arrays in solid films that change dimension in response to chemical, electrical, and thermal environmental changes. Here, we present two applications of these self-ordered colloids: (1). Photonic crystal hydrogels as trainable metal ion sensors and (2) Superparamagnetic materials.

We have utilized a soft hydrogel matrix, which contains an embedded photonic crystalline colloidal array [1], to control the microenvironments around metal ion binding sites [2]. This sensor material consists of a ca. 90\% aqueous polyacrylamide network hydrogel, which is lightly crosslinked with methylenebisacrylamide, and has pendant 8-hydroxyquinoline ligand groups (Figure 1). When this material is treated with aqueous solutions of a metal ion, the hydrogel undergoes a volume collapse, resulting in a blue shift of the Bragg diffracted wavelength of light from the embedded photonic crystal. This is due to the formation of 2:1 ligand:metal ion complex sites, ${ }^{3}$ and a rearrangement of the matrix, to create cavities around these sites. These sites are extremely robust, with very large metal:ligand affinities, as evidenced by the inability to remove the residual metal content from the gel. In addition, it is believed that the ligand bound metal ion is reduced and produces colloidal metal clusters, which stay embedded within the matrix. This hydrogel then functions as a selective optical sensor for aqueous solutions of the same metal ion, and shows only red shifts in the diffracted wavelengths upon increasing the concentration of the metal ion, due to the formation of 1:1 ligand:metal ion complexes [3]. Aqueous solutions of a different metal ion produce much smaller red shifts in this material. This effect of conservation of site architecture and hydrogel trainability to selectively bind the original metal ion is a general one, and selective sensors for $\mathrm{Zn}^{2+}$, and $\mathrm{Cu}^{2+}$, have been produced in this way. Hence this technique is an effective route to create hydrogel based sites for the selective recognition and sensing of metal ions, via control of the microenvironments around these metal ion binding sites.

To provide structural insights into the metal ion sensor mechanisms of this novel material, scanning (SEM) and transmission (TEM), including analytical, electron microscopy methods will be used. Specifically, we will investigate the position and structure of the metal (e.g. Cu) atoms within the matrix, possible oxidation states of the metal and metal interactions with the binding site, as well as the structure of the self-ordered colloidal array in the hydrogel matrix prior and after metal ion exposure.

Superparamagnetic colloid particles can also be synthesized by emulsion polymerization of styrene in the presence of nanoscale ferrite $\left(\mathrm{Fe}_{3} \mathrm{O}_{4}\right)$. The ferrites were incubated inside the $\sim 150 \mathrm{~nm}$ polystyrene particles, and appeared as black aggregates in the TEM image (Figure 2). These particles 
would self assemble into superparamagnetic photonic crystals which could be utilized as optical switch controlled by magnetic fields. Further TEM studies can provide guidelines to new synthesis methods of superparamagnetic colloidal arrays with higher magnetic moments and greater homogenous distribution.

\section{References}

[1]. (a). Asher, S. A.; Holtz, J.; Liu, L.; Wu, Z. J. Am. Chem. Soc. 1994 116, 4997-4998; (b). Holtz, J.H.; Asher, S. A. Nature 1997 389, 829-832.; (c). Holtz, J. S.; Holtz, W.; Munro, C.H.; Asher, S. A. Anal. Chem. 1998 70, 780-791.

[2]. Polymeric materials have been utilized to control the microenvironments around metal ion binding sites. See for example: Sharma, A. C.; Borovik, A. S. J. Am. Chem. Soc. 2000, 122, 8946-8955.

[3]. (a). Kim, Y-S.; Shin, J-H.; Choi, Y-S.; Lee, W.; Lee, Y-III. Microchem. J. 2001, 68, 99-107; (b). Lee, S. C.; Izzat, R. M.; Zhang, X. X.; Nelson, E. G.; Lamb, J. D.; Savage, P. B.; Bradshaw, J. S. Inorg. Chim. Acta 2001, 317, 174-180.

[4]. This research program is supported by the Department of Energy (DE-FG07-98ER62708) and Office of Naval Research (ONR) (N00014-94-1-0592).

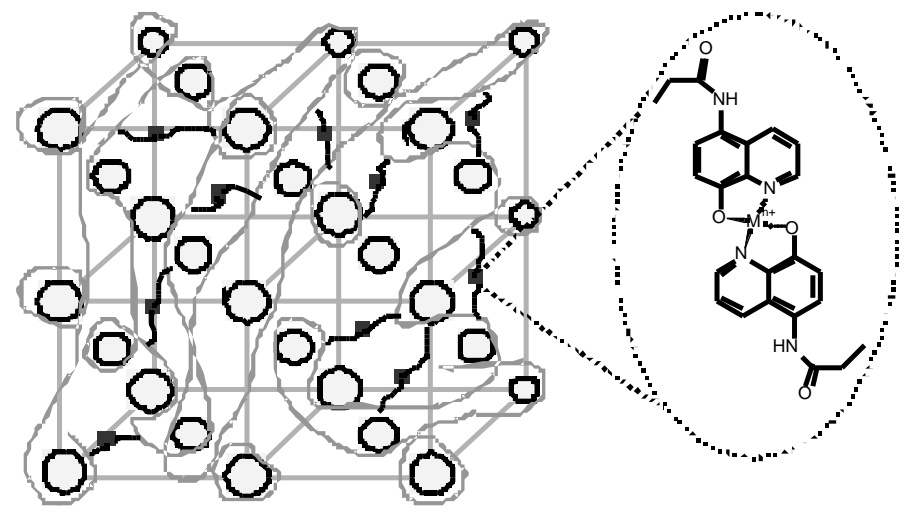

Figure 1: Illustration of the metal ion sensor material, which is a hydrogel matrix with an embedded photonic crystalline colloidal array. The enlarged diagram shows the pendant 8-hydroxyquinoline ligand group.

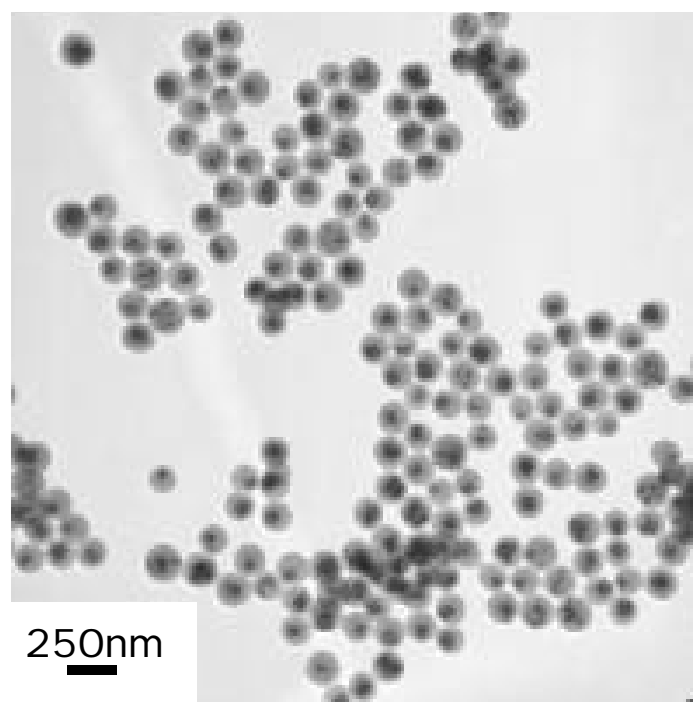

Figure 2: Bright-field image of $\mathrm{Fe}_{3} \mathrm{O}_{4}$ embedded in polystyrene as a paramagnetic material. 